\title{
Model-Based Threat Assessment in Semi-Autonomous Vehicles with Model Parameter Uncertainties
}

\author{
Mohammad Ali*†, Paolo Falcone* and Jonas Sjöberg*
}

\begin{abstract}
In this paper, we consider model-based threat assessment methods which rely on vehicle and driver mathematical models and are based on reachability analysis tools and set invariance theory. We focus on the parametric uncertainties of the driver mathematical model and show how these can be accounted for in the threat assessment. The novelty of the proposed methods lies in the inclusion of the driver model uncertainties in the threat assessment problem formulation and in their validation through experimental data. We show how different ways of accounting for the model uncertainties impact the capabilities and the effectiveness of the proposed algorithms in detecting hazardous driving situations.
\end{abstract}

\section{INTRODUCTION}

Autonomous and semi-autonomous driving technologies are increasingly gaining an important role in driver assistance systems. Since the introduction of the first electronic stability control systems [16], there has been a clear trend of increased authority of electronic systems autonomously controlling the vehicle motion. While assistance/comfort systems take the vehicle control only if requested by the driver, safety systems might take control automatically if needed. Hence, for an active safety system it is crucial to detect when it is necessary to issue an assisting intervention. The problem of determining whether a driver may need assistance, that is, when to switch from a manual to an autonomous driving mode, is in this paper referred to as threat assessment problem.

In this paper we consider a threat assessment problem in a lane guidance application. In such applications, the driver is assisted in staying within the lane through warnings and/or autonomous driving interventions. A review of various lane guidance approaches can be found in [1]. Most of the lane guidance systems in the literature keep the vehicle in the center of the lane once they have been activated and might be classified as assistance systems. In some contributions, also the transition criteria are considered [8], [10], [2]. In [8], the authors proposed that a steering intervention should drive the vehicle to a safe position in the center of the lane only if there is a risk that crossing the lane marking might lead to a collision with a vehicle in the adjacent lane. This is a restrictive approach, where many situations could be excluded in order to eliminate misuse and nuisance. In [10], the authors proposed that a lateral controller should be activated once a driver monitoring device has detected that the

\footnotetext{
*Department of Signals and Systems, Chalmers University of Technology, SE-412 96 Göteborg, Sweden, E-mail: falcone@chalmers.se, jonas.sjoberg@chalmers.se

${ }^{\dagger}$ Volvo Car Corporation, Active Safety \& Chassis, 96410/PV4A, 40531 Göteborg Sweden, E-mail: mali21@ volvocars.com
}

driver is drowsy. This is a viable option for situations where the driver is drowsy, given that driver monitoring systems are sufficiently reliable. Moreover, situations where a driver, for instance, risks loosing the vehicle control because of misevaluation of the vehicle capabilities are not considered. In [2], a transition criterion based on the limitations of the vehicle dynamics is suggested. While this reduces the risk of unmotivated interventions, the transition to autonomous mode might also be delayed too long since there might be a significant difference between the driver's limitations in controlling the vehicle and the limitations set by the vehicle dynamics.

In [11], we presented a model based method, specifically evaluating the driver's ability in safely performing a desired maneuver. In particular, assigned a driving task, we proposed a solution to the problem of evaluating whether a steering maneuver exists that $(i)$ is the output of a considered driver model and (ii) accomplishes the task while maintaining the vehicle state within a prescribed set where the driver is deemed capable of preserving the vehicle stability. We first introduce a set of constraints describing "safe" driving. Moreover, we assume an estimate of the road geometry is available over a future finite time horizon and exploit vehicle and driver modeling in order to predict future constraints violation, indicating the possibility of accident or loss of vehicle control. However, estimates of the vehicle state and the road geometry as well as vehicle and driver models are always subject to uncertainties. In this paper, we show how the threat assessment method in [11] can be extended to take model parameter uncertainties into account. We validate the proposed methods using experimental data in a roadway departure application.

The paper is organized as follows. In Section II, we provide basic definitions and results on reachability analysis and set invariance theory. In Section III, we present the vehicle and driver modeling used next in Section IV, where the threat assessment algorithm is presented. In Section V, we show how uncertainties can be included in the proposed methods, while in Section VI, we show through validation on experimental data how uncertainties influence the results provided by the proposed threat assessment algorithms. Section VII closes the paper with final remarks.

\section{Preliminaries}

We introduce a few definitions and recall basic results on set invariance theory and reachability analysis for constrained discrete-time systems. In this section we consider nominal systems only while modeling uncertainties are taken into 
account in Section V. We will denote the set of all real numbers and positive integers by $\mathbb{R}$ and $\mathbb{N}^{+}$, respectively.

Denote by $f_{a}$ the state update function of an autonomous discrete-time system

$$
x(t+1)=f_{a}(x(t)),
$$

where $x(t)$ denotes the state vector. System (1) is subject to the constraints

$$
x(t) \in \mathcal{X} \subseteq \mathbb{R}^{n},
$$

where $\mathcal{X}$ is a polyhedron. For the autonomous system (1)(2), we define the set of states that evolve to $\mathcal{S}$ in one step, as

$$
\operatorname{Pre}_{f_{a}}(\mathcal{S}) \triangleq\left\{x \in \mathcal{X} \mid f_{a}(x) \in \mathcal{S}\right\} .
$$

A set $\mathcal{O}$ is said to be a positive invariant set for the autonomous system (1) subject to the constraints in (2), if

$$
x(0) \in \mathcal{O} \Rightarrow x(t) \in \mathcal{O}, \quad \forall t \in \mathbb{N}^{+} .
$$

The set $\mathcal{O}_{\infty}$ is the maximal positive invariant set of the autonomous system (1) subject to the constraints in (2), if $0 \in \mathcal{O}_{\infty}, \mathcal{O}_{\infty}$ is a robust positive invariant set and $\mathcal{O}_{\infty}$ contains all the robust positive invariant sets contained in $\mathcal{X}$ that contain the origin.

\section{Modeling}

The threat assessment algorithm presented in Section IV is based on a standard single-track linear vehicle and a driver mathematical model, presented in [11] and reported here for completeness. For a given vehicle longitudinal speed $v_{x}$, the vehicle model can be compactly written in the following form,

$$
\dot{x}(t)=\widehat{A} x(t)+\widehat{B} u(t)+\widehat{E} w(t),
$$

where $x=\left[v_{y}, \dot{\psi}, e_{\psi}, e_{y}\right]^{T}$ and $w=\dot{\psi}_{d}$ are the state and the disturbance vectors and $u=\delta$ is the steering input command. $v_{y}$ denotes the lateral velocity, $\dot{\psi}$ is the turning rate, where $\psi$ denotes the vehicle orientation w.r.t. the fixed global frame $(X, Y)$ in Figure $1 . e_{\psi}$ and $e_{y}$ denote the vehicle orientation and position errors, respectively, w.r.t. the road centerline and $\psi_{d}$ is the desired vehicle orientation, i.e., the slope of the tangent to the curve $\Gamma_{d}$ in the point $O$. For details on the modeling and simplifying assumptions the reader is referred to [11].

\section{A. Driver model}

The literature on driver modeling is rich, see, e.g., [6] for various approaches. In the proposed threat assessment algorithm we use a low complexity driver model, inspired by the work presented in [7], [5], [15], consisting of a "pursuit" part, based on the preview of the desired path, and a correcting part, keeping the vehicle in the center of the lane.

The steering angle $\delta$ is computed as,

$$
\begin{aligned}
\delta & =K_{y} e_{y}+K_{\psi} e_{\psi}^{l p}, \\
& =K_{y} e_{y}+K_{\psi} e_{\psi}+K_{\psi} \Delta \psi_{d},
\end{aligned}
$$

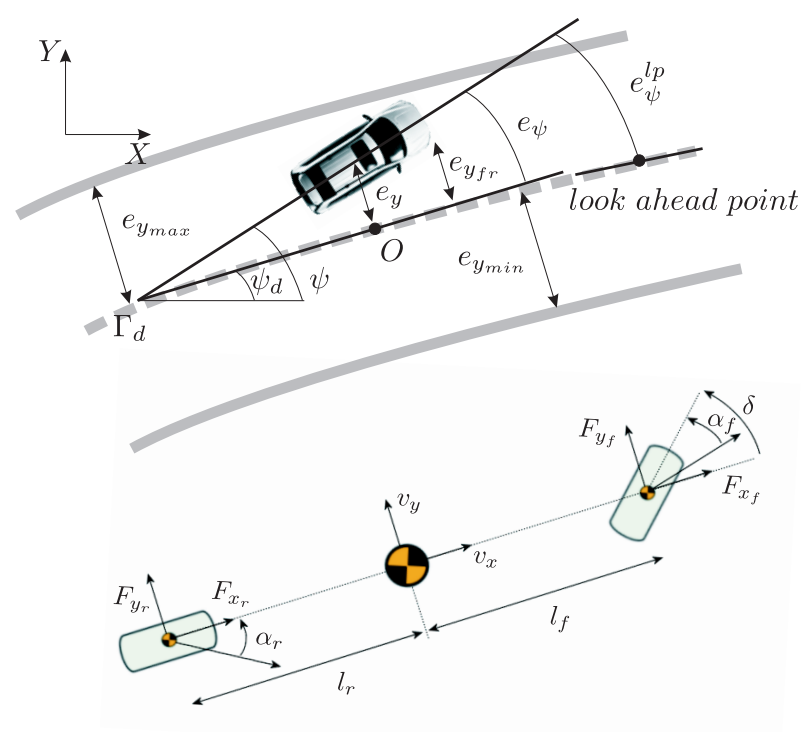

Fig. 1. Vehicle modeling notation.

where $e_{\psi}^{l p}$ is an orientation error, with respect to the orientation of the road at a distance $t_{l p} v_{x}$ ahead of the vehicle, as illustrated in Figure 1. $K_{y}, K_{\psi}$, and $t_{l p}$ are parameters of the driver model. The driver model parameters $K_{y}, K_{\psi}$, and $t_{l p}$ are in general both driver and situation dependant and might be estimated online in a vehicle application.

\section{B. Driver controlled vehicle model}

We consider the autonomous system, obtained by combining the vehicle and driver models (4) and (5), respectively, that can be compactly written as

$$
\dot{x}_{a}(t)=\widehat{A}_{a} x_{a}(t)+\widehat{E}_{a} w_{a}(t),
$$

where $x_{a}=\left[v_{y}, \dot{\psi}, e_{\psi}, e_{y}\right]^{T}$ and $w_{a}=\left[\dot{\psi}_{d}, \Delta \psi_{d}\right]^{T}$ are the state and the disturbance vectors, respectively, and the matrices $\widehat{A}_{a}, \widehat{E}_{a}$ are defined as follows,

$$
\begin{aligned}
& \widehat{A}_{a}=\left[\begin{array}{llll}
a_{11} & a_{12} & b_{1} K_{\psi} & b_{1} K_{y} \\
a_{21} & a_{22} & b_{2} K_{\psi} & b_{2} K_{y} \\
0 & 1 & 0 & 0 \\
1 & 0 & v_{x} & 0
\end{array}\right], \\
& \widehat{E}_{a}=\left[\begin{array}{ll}
0 & b_{1} K_{\psi} \\
0 & b_{2} K_{\psi} \\
-1 & 0 \\
0 & 0
\end{array}\right], \\
& a_{11}=\frac{-2\left(C_{f}+C_{r}\right)}{m v_{x}}, a_{12}=\frac{-2\left(C_{f} l_{f}-C_{r} l_{r}\right)}{m v_{x}}-v_{x}, \\
& a_{21}=\frac{-2\left(C_{f} l_{f}-C_{r} l_{r}\right)}{J_{z} v_{x}}, a_{22}=\frac{-2\left(C_{f} l_{f}^{2}+C_{r} l_{r}^{2}\right)}{J_{z} v_{x}}, \\
& b_{1}=\frac{2 C_{f}}{m}, b_{2}=\frac{2 C_{f} l_{f}}{J_{z}},
\end{aligned}
$$

where $m$ and $J_{z}$ denote the vehicle mass and yaw inertia, respectively, $l_{f}$ and $l_{r}$ are the distances of the vehicle center of gravity from the front and rear axles, respectively and $C_{f}$, $C_{r}$ denote the front and rear cornering stiffness respectively. 


\section{System Constraints}

Next we define a set of operating conditions, in the space of the states and inputs of systems (4) and (6), corresponding to stable driving within the lane boundaries.

Constraints on vehicle position are set by the limited lane width. We denote by $e_{y_{i j}}, i \in\{f, r\}, j \in\{l, r\}$, the distances of the four vehicle corners from the lane centerline. By assuming small orientation errors, $e_{y_{i j}}$ can be written as

$$
\begin{aligned}
& e_{y_{f l}}=e_{y}+\frac{c}{2}+a e_{\psi}, \\
& e_{y_{r l}}=e_{y}+\frac{c}{2}-b e_{\psi}, \quad e_{y_{f r}}=e_{y}-\frac{c}{2}+a e_{\psi}, \\
& y_{r r}=e_{y}-\frac{c}{2}-b e_{\psi},
\end{aligned}
$$

where $c$ is the vehicle width, $a$ and $b$ are the distances of the center of gravity from the front and rear vehicle bumpers, respectively.

Furthermore, in order to avoid possible vehicle instability due to the effects of the tire nonlinearities the vehicle can be forced to operate in the linear region of the state space by limiting the tire slip angles $\alpha_{i}, i \in\{f, r\}$. In this region a normal driver is deemed capable of controlling the vehicle [11].

The constraints on the vehicle position and slip angles can be written as

$$
\begin{aligned}
-e_{y_{\max }} & \leq e_{y_{i j}} \leq e_{y_{\max }}, \\
\alpha_{i_{\min }} & \leq \alpha_{i} \leq \alpha_{i_{\max }}, i \in\{f, r\}, j \in\{l, r\},
\end{aligned}
$$

where $e_{y_{\max }}$ is the maximum distance of the vehicle corners from the lane centerline. The constraints (9) can then be compactly rewritten as

$$
H_{a} x_{a} \leq h_{a}
$$

\section{SET-BASED THREAT ASSESSMENT}

We discretize the model (6) with a sampling time $T_{s}$, to obtain the discrete time constrained autonomous system subject to disturbances

$$
\begin{array}{cl}
x_{a}(t+1)= & A_{a}^{d} x_{a}(t)+E_{a}^{d} w_{a}(t) \\
\text { subj. to } \quad & H_{a} x_{a}(t) \leq h_{a},
\end{array}
$$

where, for the sake of simple notation, we have denoted the state, the disturbance and the time index with the same symbols as in (6).

We introduce the following assumptions on the disturbance signal $w_{a}$,

Assumption 1: $w_{a}(t) \in \mathcal{W}, \forall t \geq 0$, where $\mathcal{W} \subseteq \mathbb{R}^{2}$ is a polyhedron.

Assumption 2: Every time instant $t$, the disturbance $w_{a}(t)$ is known over a finite time horizon of $N$ steps.

Assumption 2 implies that the road geometry is known over a future time horizon. The sensing technologies used in, e.g., [9], [12], [4] can be used for this purpose.

\section{A. Main Algorithm}

We denote by $\mathcal{X}_{\text {feas }}$ the set of admissible states,

$$
\mathcal{X}_{\text {feas }}=\left\{x_{a} \in \mathbb{R}^{4}: H_{a} x_{a} \leq h_{a}\right\} .
$$

Every time instant, we consider a terminal target set $\mathcal{T} \subseteq$ $\mathcal{X}_{\text {feas }}$. Further details about the choice of $\mathcal{T}$ are provided in [11]. Denote by $W_{t}=\left[w_{t}, w_{t+1}, \ldots, w_{t+N-1}\right]$, the sequence of disturbance samples over the time horizon $[t, t+N-1]$ and by $W_{t, i}=\left[w_{t+i}, \ldots, w_{t+N-1}\right]$ any sequence extracted from $W_{t}$. We compute the sequence of states sets $X_{t}\left(W_{t}\right)=$ $\left[\mathcal{X}_{t}, \mathcal{X}_{t+1}, \ldots, \mathcal{X}_{t+N-1}\right]$ as:

$$
\begin{array}{cr}
\mathcal{X}_{t+i}\left(W_{t, i}\right) & =\mathcal{X}_{\text {feas }} \bigcap \operatorname{Pre}_{f_{a}}\left(\mathcal{X}_{t+i+1}, w_{t+i}\right), \\
\mathcal{X}_{t+N} & =\mathcal{T},
\end{array}
$$

where $f_{a}$ denotes the right hand side of (11a). We call the set $\mathcal{X}_{t}$ the safe set at time $t$.

We observe that the calculation of the sequence $X_{t}\left(W_{t}\right)$ is performed every time step, based on the updated disturbance sequence $W_{t}$. Moreover, if at the current time $t$ the state of the system (11) belongs to the safe set $\mathcal{X}_{t}$, the autonomous system (11), i.e., the vehicle in closed loop with the driver, is guaranteed to evolve to the set $\mathcal{T}$ in $N$ steps, while satisfying the constraints (11b). The algorithm is outlined in

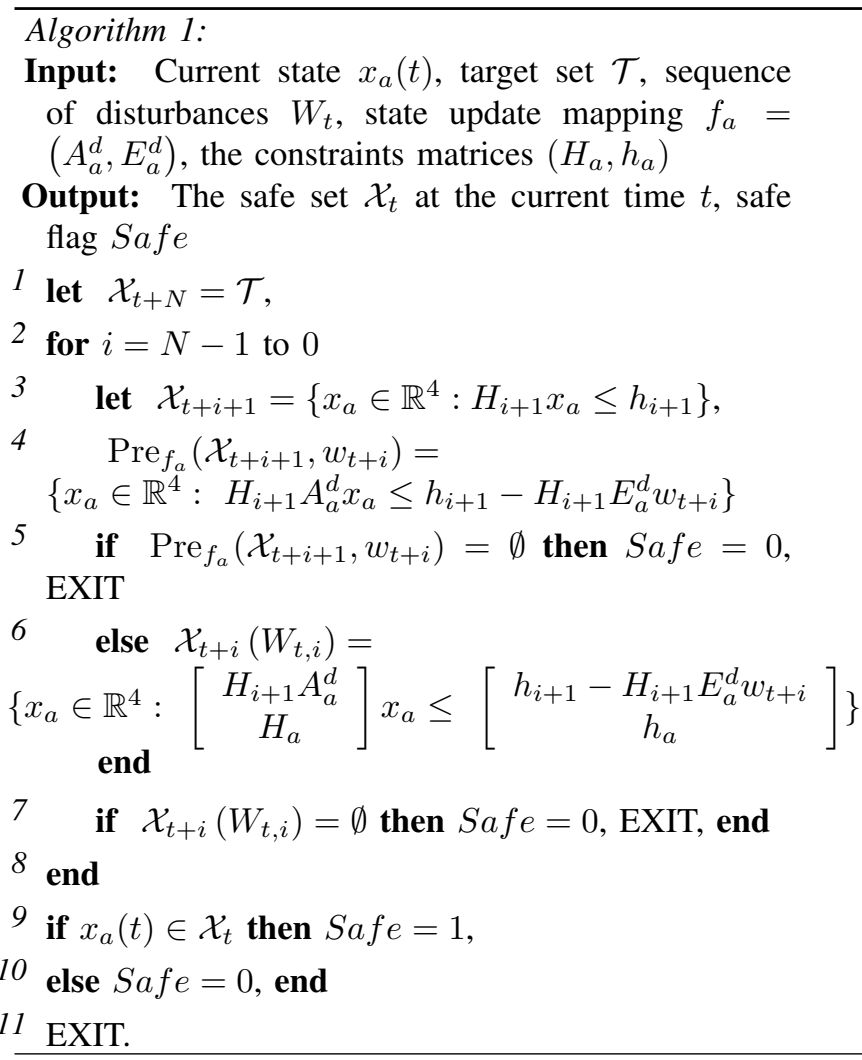

detail in Algorithm 1, where the inputs are: the current state $x_{a}(t)$, the target set $\mathcal{T}$, the sequence of disturbances $W_{t}$, the state update mapping $f_{a}=\left(A_{a}^{d}, E_{a}^{d}\right)$, the constraints matrices $\left(H_{a}, h_{a}\right)$, and the outputs are: the safe set $\mathcal{X}_{t}$ at the 
current time $t$ and the flag Safe which takes the value 0 if an intervention is needed and 1 otherwise.

\section{MOdeling UnCERTAinty}

Algorithm 1 does not account for any uncertainties in the vehicle and driver model or measurements. Next, we present alternative strategies accounting for model parameter uncertainties. We assume that the parameters of the matrices $A_{a}^{d}, E_{a}^{d}$ in (11) are subject to polytopic uncertainty according to the following,

Assumption 3: $A_{a}^{d} \in \mathcal{A}$ and $E_{a}^{d} \in \mathcal{E}$ where,

$$
\begin{aligned}
& \mathcal{A}=\left\{A_{a}^{d} \in \mathbb{R}^{n \times n} \mid A_{a}^{d}=\sum_{i=1}^{N_{A}} \theta_{i} \tilde{A}_{i}, \sum_{i=1}^{N_{A}} \theta_{i}=1, \theta_{i} \geq 0\right\} \\
& \mathcal{E}=\left\{E_{a}^{d} \in \mathbb{R}^{n \times p} \mid E_{a}^{d}=\sum_{i=1}^{N_{A}} \theta_{i} \tilde{E}_{i}, \sum_{i=1}^{N_{A}} \theta_{i}=1, \theta_{i} \geq 0\right\} . \\
& \text { Definition } 1 \text { (Parameter Underestimated Pre set): }
\end{aligned}
$$
Denote by,

$$
\operatorname{Pre}_{f_{a}}^{\mathrm{PU}}(\mathcal{S}) \triangleq \bigcap_{A_{a}^{d} \in \mathcal{A}, E_{a}^{d} \in \mathcal{E}} \operatorname{Pre}_{\left(A_{a}^{d}, E_{a}^{d}\right)}(\mathcal{S}),
$$

the set of states for which the system (6) evolves to $\mathcal{S}$ in one step $\forall A_{a}^{d} \in \mathcal{A}, E_{a}^{d} \in \mathcal{E}$.

The set $\operatorname{Pre}_{f_{a}}^{\mathrm{PU}}(\mathcal{S})$ is the largest set of states which can be guaranteed to be enclosed by $\operatorname{Pre}_{\left(A_{a}^{d}, E_{a}^{d}\right)}(\mathcal{S})$ under Assumption 3. However, the computation of $\operatorname{Pre}_{f_{a}}^{\mathrm{PU}}(\mathcal{S})$ through the relation (14) requires an infinite intersection of sets, we therefore introduce the following

Proposition 1:

$$
\operatorname{Pre}_{f_{a}}^{\mathrm{PU}}(\mathcal{S})=\bigcap_{i=1}^{N_{A}} \operatorname{Pre}_{\left(\tilde{A}_{i}, \tilde{E}_{i}\right)}(\mathcal{S}) .
$$

According to Proposition 1, only a finite intersection of sets is required in order to calculate the set $\operatorname{Pre}_{f_{a}}^{\mathrm{PU}}(\mathcal{S})$. The proof of Proposition 1 follows immediately from the proof provided in [14] for hybrid systems.

Definition 2 (Parameter Overestimated Pre set): Denote by,

$$
\operatorname{Pre}_{f_{a}}^{\mathrm{PO}}(\mathcal{S}) \triangleq \bigcup_{A_{a}^{d} \in \mathcal{A}, E_{a}^{d} \in \mathcal{E}} \operatorname{Pre}_{\left(A_{a}^{d}, E_{a}^{d}\right)}(\mathcal{S})
$$

the set of states that evolve to $\mathcal{S}$ in one step for some model $A_{a}^{d} \in \mathcal{A}, E_{a}^{d} \in \mathcal{E}$.

The set $\operatorname{Pre}_{f_{a}}^{\mathrm{PO}}(\mathcal{S})$ is the smallest set of states which can be guaranteed to enclose $\operatorname{Pre}_{\left(A_{a}^{d}, E_{a}^{d}\right)}(\mathcal{S})$ under Assumption 3.

Remark 1: We observe that the relation (16), is an infinite union of polyhedral sets and that, in general, the union of polyhedral sets can not be guaranteed to be a convex set [3]. The convex hull Conv $\left(\operatorname{Pre}_{f_{a}}^{\mathrm{PO}}(\mathcal{S})\right)$ is the smallest convex set enclosing $\operatorname{Pre}_{f_{a}}^{\mathrm{PO}}(\mathcal{S})$.

We are interested in finding a polyhedral set, that can be computed through a finite number of evaluations and, in addition, encloses $\operatorname{Pre}_{f_{a}}^{\mathrm{PO}}(\mathcal{S})$. In general, a relation corresponding to Proposition 1 does not hold for (16). However, the set Conv $\left(\operatorname{Pre}_{f_{a}}^{\mathrm{PO}}(\mathcal{S})\right)$ can be approximated by gridding $\mathcal{A}$ and evaluating a finite union of polytopes.

\section{A. Modified Algorithms}

Denote by $\mathcal{X}_{t,\left(A_{a}^{d}, E_{a}^{d}\right)}$ the safe set at time $t$ obtained through the recursion (13). Let

$$
\mathcal{X}_{t}^{\mathrm{PU}}=\bigcap_{A_{a}^{d} \in \mathcal{A}, E_{a}^{d} \in \mathcal{E}} \mathcal{X}_{t,\left(A_{a}^{d}, E_{a}^{d}\right)},
$$

and

$$
\tilde{\mathcal{X}}_{t}^{\mathrm{PO}}=\operatorname{Conv}\left(\mathcal{X}_{t}^{\mathrm{PO}}\right)=\operatorname{Conv}\left(\bigcup_{A_{a}^{d} \in \mathcal{A}, E_{a}^{d} \in \mathcal{E}} \mathcal{X}_{t,\left(A_{a}^{d}, E_{a}^{d}\right)}\right),
$$

denote the model parameter under- and overestimated safe sets at time $t$, respectively. Replacing $\mathcal{X}_{t}$ in step 9 of Algorithm 1 by the set $\mathcal{X}_{t}^{\mathrm{PU}}$ results in an algorithm which, for the assumed vehicle and driver models, guarantees the detection of a constraint violation occurring within the future $N$-steps. As a consequence, this approach might lead to an increased frequency of false risk detection. Replacing $\mathcal{X}_{t}$ with $\tilde{\mathcal{X}}_{t}^{\mathrm{PO}}$ instead, guarantees that, according to the assumed vehicle and driver models, no false risk detections can occur at the cost of decreased capability of detecting accident risk.

We will refer to the algorithm obtained by replacing $\mathcal{X}_{t}$ in step 9 of Algorithm 1 by the set $\mathcal{X}_{t}^{\mathrm{PU}}$, as Algorithm 2, and the algorithm obtained by replacing $\mathcal{X}_{t}$ by $\tilde{\mathcal{X}}_{t}^{\text {PO }}$, as Algorithm 3.

Remark 2: In order to ensure convexity (see Remark 1), we have used $\tilde{\mathcal{X}}_{t}^{\mathrm{PO}}$ instead of $\mathcal{X}_{t}^{\mathrm{PO}}$ in Algorithm 3. In general, compared to $\mathcal{X}_{t}^{\mathrm{PO}}$ this is a further overestimation of the safe set and might further decrease the capability of detecting accident risk.

\section{RESUlts}

In this section, we consider the driver model parameters $K_{y}$ and $K_{\psi}$ as uncertain and validate the results obtained with the present approaches on experimental data. The parameters $K_{y}, K_{\psi}$ and $t_{l p}$ can be estimated online using a recursive nonlinear least squares method [13]. At each time step, an estimate of the mean and variance is updated as new data becomes available. For the experimental validation of the parameter estimation and threat assessment results presented next, data has been collected on a test track resembling a country road and post-processed offline.

Figure 2 depicts the ellipsoidal set, defined by the estimated covariance matrix, where the uncertain parameters $K_{y}$ and $K_{\psi}$ lie. In Algorithm 1 we use the estimated mean of the parameters $K_{y}, K_{\psi}$ and $t_{l p}$, while in Algorithms 2-3, we use the estimated mean of $t_{l p}$ and treat the parameters $K_{y}$ and $K_{\psi}$ as uncertain. In particular, every time step we approximate the ellipsoidal set in Figure 2 as a polyhedral set $\mathcal{K}$. Figure 2, shows an example where only four vertices have been used for the polyhedral approximation. A more accurate approximation can of course be obtained by increasing the number of vertices.

Let the pair $\tilde{A}_{i}, \quad \tilde{E}_{i}$ be the matrices in (7), where the values of $K_{y}$ and $K_{\psi}$ are set by their corresponding values at vertex $i$ of $\mathcal{K}$ and with values of the rest of the parameters according to Table I. In the following results, the set $\mathcal{K}$ is computed with four vertices only hence the sets $\mathcal{A}, \mathcal{E}$, are defined as convex combinations of the discretized matrices 


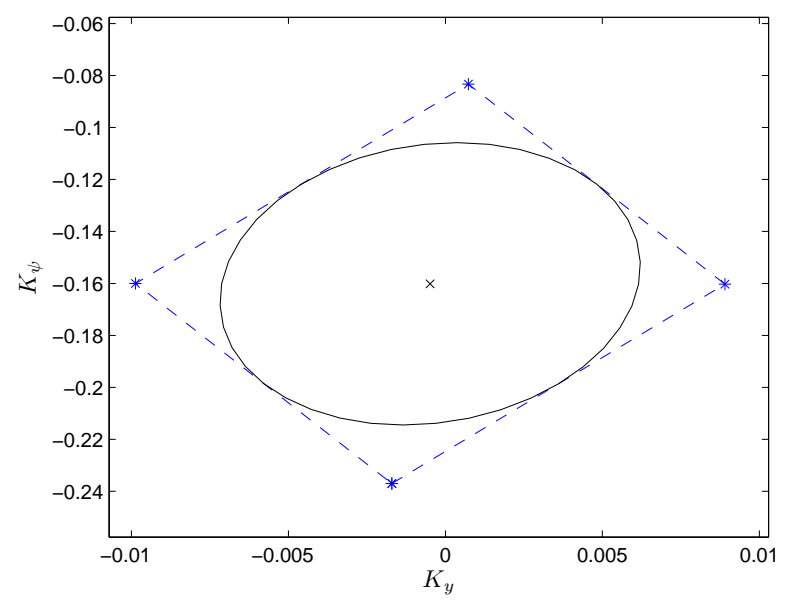

Fig. 2. Estimation of the driver model parameters $K_{y}$ and $K_{\psi}$. The $\times$ denotes the estimated mean, the ellipse denotes the variance, the dashed box denotes a polyhedral approximation $\mathcal{K}$ of the variance and the $*$ denotes the vertices of $\mathcal{K}$.

TABLE I

VEHICLE PARAMETERS

\begin{tabular}{|c|c|c|c|c|}
\hline$m$ & $J_{z}$ & $l_{f}$ & $l_{r}$ & $a$ \\
\hline $1695 \mathrm{~kg}$ & $2617 \mathrm{kgm}^{2}$ & $1.14 \mathrm{~m}$ & $1.50 \mathrm{~m}$ & $1.83 \mathrm{~m}$ \\
\hline$C_{f}$ & $C_{r}$ & $b$ & $c$ & \\
\hline $54 \mathrm{kNm} / \mathrm{rad}$ & $45 \mathrm{kNm} / \mathrm{rad}$ & $2.69 \mathrm{~m}$ & $1.77 \mathrm{~m}$ & \\
\hline
\end{tabular}

$\tilde{A}_{1}^{d}, \ldots, \tilde{A}_{4}^{d}, \tilde{E}_{1}^{d}, \ldots, \tilde{E}_{4}^{d}$, respectively. In addition, the following numerical values have been set,

$$
\begin{aligned}
& \alpha_{f_{\text {max }}}=\alpha_{r_{\text {max }}}=-\alpha_{f_{\text {min }}}=-\alpha_{r_{\text {min }}}=4^{\circ}, \\
& e_{y_{\text {max }}}=1.56 \mathrm{~m}, N=10, T_{s}=50 \mathrm{~ms}, \mathcal{T}=\mathcal{X}_{\text {feas }} .
\end{aligned}
$$

Algorithm 2 guarantees that interventions are issued if a constraint violation is imminent for the estimated range of possible values of the driver parameters and is thus the safest approach. Algorithm 3 guarantees that no unnecessary interventions are issued since it waits until none of the driver models in the estimated range can avoid a constraint violation. From a nuisance point of view, Algorithm 3 might be preferable. Algorithm 1 can be considered as a trade-off between the two.

In practice, decisions to intervene will be issued at different time instances by the three algorithms. Of course, a difference in decisions between the algorithms does not necessarily imply that any of the algorithms decision is incorrect. In order to compare the algorithms we will however introduce a unified criteria for classifying interventions. Denote by $t^{*}$ a time instant where $x$ exits the set $\mathcal{X}_{\text {feas }}$ and let $T^{*}=\left[t^{*}-(N+\epsilon) T_{s}, t^{*}-(N-\epsilon) T_{s}\right]$ be a time interval where a decision to intervene is expected. We consider decisions to intervene that occur in a time interval $T^{*}$ as correct, decisions that occur prior to $T^{*}$ early interventions and decision that occur between $T^{*}$ and $t^{*}$ late interventions. Completely missed interventions are classified as late.

The performance of the three algorithms has been evalu-

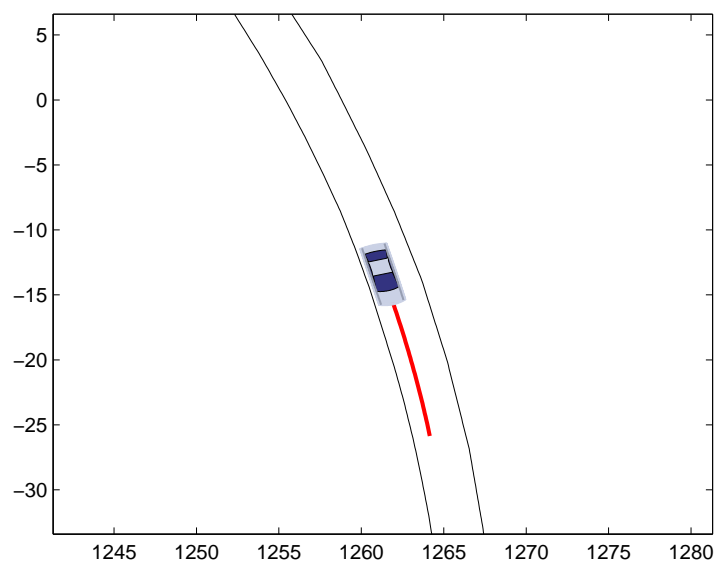

Fig. 3. Illustration of a situation where a vehicle is negotiating a curve. The situation is particularly challenging since the vehicle is very close to the road edge. The solid line originates at the current state and shows the vehicle's future trajectory over horizon of $N$-steps.

ated on approximately $20 \mathrm{~km}$ of data and the results for $\epsilon=2$ are shown in Table II.

TABLE II

PERFORMANCE ON APPROXIMATELY 20KM DATA

\begin{tabular}{|c|c|c|}
\hline Algorithm & Early intervention & Late intervention \\
\hline 1 & 17 & 6 \\
\hline 2 & 32 & 4 \\
\hline 3 & 4 & 6 \\
\hline
\end{tabular}

We observe that Algorithm 2 tends to issue interventions early. Sometimes interventions are issued even when no constraint violation is imminent in order to guarantee safety. To some extent also Algorithm 1 tends to issue early interventions but the frequency is much lower than for Algorithm 2. Algorithm 3 waits until a constraint violation is unavoidable for all of the considered values of the driver model parameters. This reduces the number of early interventions significantly. One might suspect that this would lead to an increase in the number of late interventions instead (see Remark 2), but the validation results do not show any significant increase. In fact, through inspection of the late interventions, we have observed that, in all cases, the subsequent constraint violation consisted of a short drift out of the lane of less than $10 \mathrm{~cm}$. Such constraint violations, are typically not very dangerous, but difficult to predict.

Figure 3 shows a situation that is particularly challenging. The vehicle is driving very close to the road border and might cross it very fast. The vehicle speed is however not so high that the vehicle dynamics prohibit the possibility to stay on the road. Figures 4-5 show the safe sets obtained in the illustrated situation. In this situation $\mathcal{X}_{t}^{P U}$ is a very small set and does not enclose the measured state. Hence, according to Algorithm 2, a driver exists, modeled by (5) with parameters lying in the set $\mathcal{K}$ (See Figure 2), who 


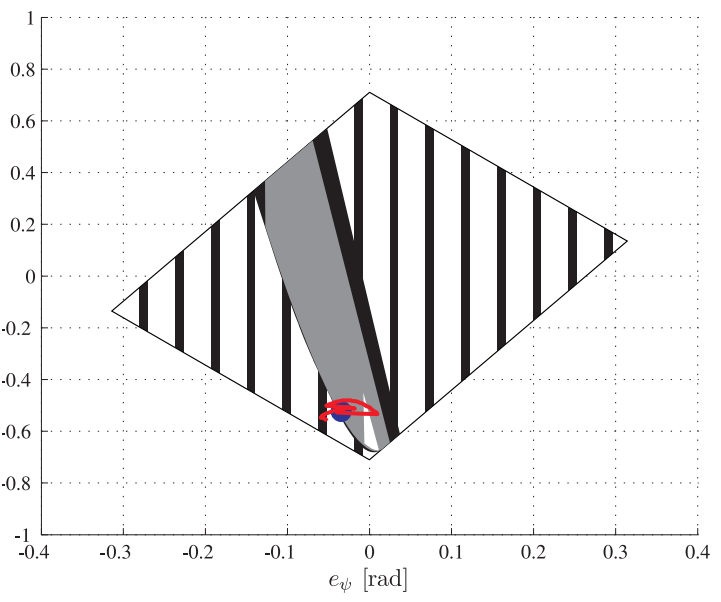

Fig. 4. Illustration of the obtained safe sets. The small white set denotes $\mathcal{X}_{t}^{P U}$, the grey set denotes $\mathcal{X}_{t}$, the black set denotes $\tilde{\mathcal{X}}_{t}^{P O}$ and the striped set denotes $\operatorname{Conv}\left(\bigcup_{A \in \mathcal{A}, E \in \mathcal{E}} \mathcal{X}_{\text {feas },(A, E)}\right)$. The blue circle denotes the measured state and the solid line originates at the current state and shows the vehicle's future trajectory over the horizon of $N$-steps. The sets are cuts shown in the $e_{y}-e_{\psi}$ space evaluated at the measured values of $v_{y}, \dot{\psi}$.

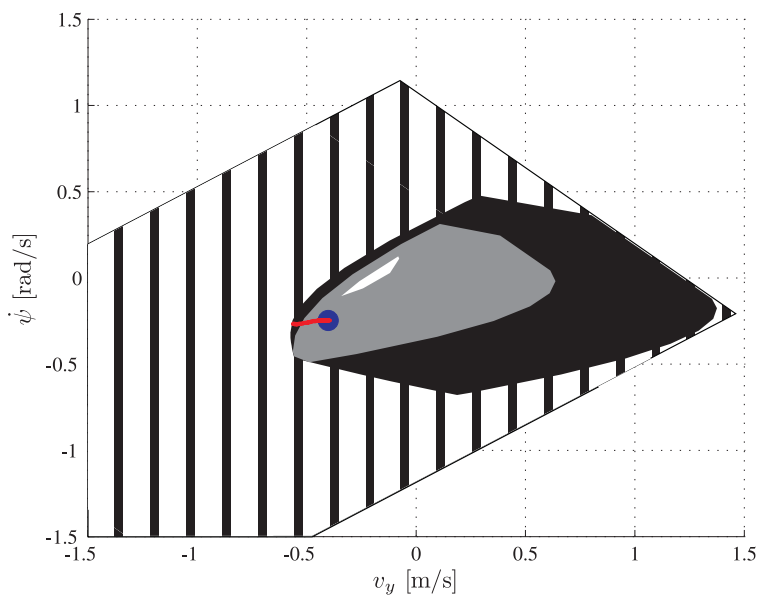

Fig. 5. Illustration of the obtained safe sets. The color convention in Figure 4 has been used. The sets are cuts shown in the $v_{y}-\dot{\psi}$ space evaluated at the measured values of $e_{y}, e_{\psi}$

cannot keep the vehicle on the road and as, a consequence, an intervention is needed. The set $\mathcal{X}_{t}$ is larger than $\mathcal{X}_{t}^{P U}$ and, in fact, encloses the measured state. According to Algorithm 1, the driver model (5) where the parameters are set by the estimated mean, is capable of keeping the vehicle state within $\mathcal{X}_{\text {feas }}$ over the horizon of $N$-steps. This is in fact confirmed by the solid line, originating at the current state and showing the future trajectory of the vehicle over the horizon. The set $\tilde{\mathcal{X}}_{t}^{P O}$ is even larger and hence also encloses the measured state. According to $\tilde{\mathcal{X}}_{t}^{P O}$, even though some of the considered driver models will drive the vehicle outside the lane at least one is capable of keeping the vehicle within $\mathcal{X}_{\text {feas }}$. According to Algorithm 3, the situation should thus be considered safe and no intervention should be issued.

\section{CONCLUSIONS AND FUTURE WORKS}

We have presented model based threat assessment methods for semi-autonomous vehicles and studied the impact of model parameter uncertainties on the capability of detecting hazardous driving situations. The obtained results demonstrate that the proposed methods can effectively predict lane crossing and vehicle instability over a future, finite time horizon, thus allowing the activation of lower level control interventions. Depending on how model parameter uncertainties are treated the interventions will be activated at different points in time, affecting the performance of the algorithms. The preliminary results presented in this manuscript indicate that, compared to the other algorithms, Algorithm 3 reduces the number of early interventions significantly while maintaining the ability to predict constraint violations. Consequently, computationally efficient modifications of Algorithm 3 is currently the topic of ongoing research efforts. In addition further investigations aiming at analyzing the impact of measurement errors on the vehicle state and disturbance signals originating from e.g. uncertainty in the sensing of the road geometry will be conducted.

\section{REFERENCES}

[1] M Ali. On Automotive Roadway Departure Prevention. Licentiate thesis, Chalmers University, 2010.

[2] S J Anderson, S C Peters, T E Pilutti, and K D Iagnemma. An Optimal-Control-Based Framework for Trajectory Planning, Threat Assessment, and Semi-Autonomous Control of Passenger Vehicles in Hazard Avoidance Scenarios: Experimental Results. In Proc. Conference on Field and Service Robots, 2009.

[3] A Bemporad. Convexity recognition of the union of polyhedra. Computational Geometry, 18(3):141-154, April 2001.

[4] M Bertozzi, A Broggi, and A Fascioli. Vision-based intelligent vehicles: State of the art and perspectives. Robotics and Autonomous Systems, 32(1):1-16, July 2000.

[5] F. Braghin, F. Cheli, S. Melzi, and E. Sabbioni. Race driver model. Computers and Structures, 86:1503-1516, 2008.

[6] C Cacciabue. Modelling Driver Behaviour in Automotive Environments. Springer, 2007.

[7] D. Casanova. On Minimum Time Vehicle Manoeuvring: The theoretical Optimal Lap. Phd thesis, Mechanical Engineering, Cranfield University, 2000.

[8] A Eidehall and F Gustafsson. A new approach to lane guidance systems. In Proc. IEEE Conference on Intelligent Transportation System, 2005

[9] A Eidehall, J Pohl, and F Gustafsson. Joint road geometry estimation and vehicle tracking. Control Engineering Practice, 2007.

[10] N M Enache, M Netto, and B Lusetti. Driver Steering Assistance for Lane-Departure Avoidance Based on Hybrid Automata and Composite Lyapunov Function. IEEE Transactions on Intelligent Transportation Systems, pages 1-12, 2009.

[11] P Falcone, M Ali, and J Sjöberg. Predictive Threat Assessment via Reachability Analysis and Set Invariance Theory. IEEE Transactions on Intelligent Transportation Systems, 12(3), July 2011.

[12] J Jansson. Collision avoidance theory with application to automotive collision mitigation. PhD thesis, 2005.

[13] S M Kay. Fundamentals of statistical signal processing: estimation theory. Prentice hall signal processing series, 1993.

[14] H Lin, X D Koutsoukos, and P J Antsaklis. Hierarchical Control for a Class of Uncertain Piecewise Linear Hybrid Dynamical Systems. In Proceedings of the 2002 IFAC 15th World Congress, Barcelona, 2002.

[15] H. Peng and M. Tomizuka. Preview Control for Vehicle Lateral Guidance in Highway Automation. Journal of Dynamic Systems, Measurement, and Control, 115, 1993.

[16] A T Van Zanten, R Erhardt, and G Pfaff. VDC, The Vehicle Dynamics Control System of Bosch. SAE, 1995. 